\section{PTU-198 SERUM FGF19 LEVELS ARE RELATED TO DISEASE ACTIVITY IN ILEAL CROHN'S DISEASE}

doi:10.1136/gutjnl-2013-304907.288

1."J D Nolan, II Johnston, ${ }^{2 T}$ Dew, 'S Pattni, ' J R Walters. 'Gastroenterology, Imperial College London; ${ }^{2}$ Biochemistry, Kings College London, London, UK

Introduction FGF19 is a polypeptide hormone produced in the ileum. It's transcription is stimulated by bile acid (BA) activation of the Farnesoid X receptor (FXR). In human cell lines the transcription of FGF19 is suppressed by pro inflammatory cytokines (TNF- $\alpha$, IL-1 $\beta$ ) via inhibitory effects on 'FXR'. We hypothesised that circulating FGF19 levels will be low in patients with active ileal Crohn's disease and that these levels will increase after the administration of anti-inflammatory treatments commonly used to induce clinical remission.

Methods Fasting circulating FGF19 levels were measured using ELISA. Disease activity was assessed using the Harvey Bradshaw index. In a cross sectional study, 30 patients with Crohn's disease involving the ileum (and no previous intestinal resections) were recruited, 12 with active disease $(\mathrm{HBI}>4)$ and 8 with inactive disease. In longitudinal studies, 4 patients with active colonic disease and 5 patients with active disease involving the ileum had samples taken whilst their disease was active and again during medically induced clinical remission ( $\mathrm{HBI}<4$ induced by corticosteroids or anti-TNF) over 6 weeks later. 6 patients with CD involving the ileum ( 5 with previous ileal resection) whose disease remained inactive, had FGF19 levels samples measured taken on 2 separate occasions over 6 weeks apart. Non parametric statistical tests were used (Mann whitney, medians and ranges shown).

Results In the cross sectional study serum FGF19 levels were significantly lower in those patient with CD involving the ileum with active disease (median 40, 3- 338) compared to inactive disease (median 147, 46-255, $\mathrm{p}=0.0486$ ). In the longitudinal studies, serum FGF19 levels were significantly lower in the patients with CD involving the ileum whilst their disease was active (median 35, range $3-54$ ) compared to paired levels taken after medically induced remission (median 141, range 116 - 184, $p=0.002$ ). Paired FGF19 levels were not statistically different in the 4 patients with colonic Crohn's disease during active disease (median 105, range 36 - 151) compared to medically induced clinical remission (median 86, range $40-120, p=0.48$ ). There was no significant difference in paired FGF19 levels In the 6 patients with CD involving the ileum with inactive disease taken on 2 seperate occasions ( 5 with previous ileal resections).

Conclusion Circulating FGF19 levels are lower during active ileal or ileo-colonic crohn's disease and increase after medically induced clinical remission. FGF19 levels may be induced by anti-inflammatory treatments either as a result of the inhibition of inflammtory cytokines within the ileum or due to enhanced BA absorption post treatment.

Disclosure of Interest None Declared.

\section{PTU-199 PROINFLAMMATORY CYTOKINE (IL-8) IN MICROSCOPIC ENTERITIS}

doi:10.1136/gutjnl-2013-304907.289

1,"K Rostami, ${ }^{2 \mathrm{M}}$ Rostami Nejad, ${ }^{2} \mathrm{H}$ Asadzadeh, ${ }^{2 \mathrm{M}} \mathrm{R}$ Zali. ${ }^{1}$ Department of Gastroenterology, Luton \& Dunstable Hospital NHS Foundation Trust, Luton, Lotun, UK; ' ${ }^{2}$ Gastroenterology and Liver Diseases Research Center, Shahid Beheshti University of Medical Sciences, Tehran, Iran, Islamic Republic of

Introduction Celiac disease (CD) is an autoimmune disorder that is triggered by an immune response to gluten in genetically predisposed individuals. Interleukin-8 (IL-8) is produced by macrophages, epithelial cells and endothelial cells. Therefore, IL8 has an important role in the innate immune response and associated with inflammation. The aim of this study is to evaluate the elevation of IL8 level in patients with celiac diseasecompare with control group.
Methods Fifty three confirmed treated or untreated celiac disease patients referred to Taleghani hospital, Iran as cases were matched according to age and gender with 300 healthy controls, screened with ELISA to determine the level of IL8.

Results Serum IL8 levels were evaluated in all patients with positive CD compare to healthy control. The minimum level of IL8 in cases was 16.3 and maximum level was 397.7. On the other side the minimum and maximum level of IL8 in controls was 10.3 and 397.7 respectively. The mean level of IL8 in celiac disease patients was 116.8 compare with 84.8 in healthy control. This differences was statistically significant between 2 groups $(p=0.0001)$.

Conclusion Increased serum levels of pro-inflammatory cytokines such as IL8 seem to be increased in patients with celiac disease. It is the inflammation inherent in gluten sensitivity rather than mucosal atrophy that results in malabsorption and nutrient deficiency. Our findings suggest that higher level of IL8 in patients with celiac disease characterise the higher inflammatory response of this disease in the active phase. The inflammatory response including increasing the level of IL-8 may cause or not damage to the intestinal mucosa but in both cases lead to increased permeability of the intestinal epithelium. Disclosure of Interest None Declared

\section{Poster presentation II}

\section{Colorectal/Anorectal}

\section{PWE-001 THE IMPACT OF COLONIC RESECTION FOR NEOPLASIA ON FUNCTIONAL OUTCOMES AND RELATED OUALITY OF LIFE: A CASE-CONTROLLED STUDY}

doi:10.1136/gutjnl-2013-304907.290

${ }^{1, *} \mathrm{~A}$ Brigic, ${ }^{2} \mathrm{~S}$ Sakuma, ${ }^{3 \mathrm{R}}$ Lovegrove, 'S K Clark, ${ }^{2 \mathrm{P}}$ Bassett, ${ }^{3} \mathrm{~N} \mathrm{~J}$ Mortensen, 'R H Kennedy. 'Department of Surgery, St. Mark's Hospital and Academic Institute, ${ }^{2}$ Department of Research and Development, North West London Hospitals Trust, London; ${ }^{3}$ Department of Surgery, Oxford Radcliffe Hospitals, Oxford, UK

Introduction Aim Functional outcomes after elective colectomy (right, left or sigmoid) for neoplasia are poorly reported in the literature. This study evaluates bowel function and related quality of life in patients $2-4$ years after hemicolectomy, comparing the results to healthy controls.

Methods Eligible patients were identified from prospectively maintained colorectal databases at two teaching hospitals. Patients were recruited during regular follow up visits or by telephone interview. Patient relatives with an intact colon were recruited as controls. Study exclusion criteria were poor cognitive ability, inflammatory bowel disease, metastatic disease, previous pelvic radiation, prior colonic, stomach or small bowel resection, bypass surgery, vagotomy, an existing stoma and anal incontinence. The Memorial Sloan-Kettering Cancer Centre (MSKCC) questionnaire was used to assess bowel function and the EQ-5D questionnaire to assess quality of life (QOL). Parametric and non-parametric tests were used with log transformation and regression methods for skewed data. Association between EQ-5D and MSKCC scores was examined with Spearman's rank correlation.

Results A total of 85 patients (mean age $69+/-11.2$ years) and 85 controls (mean age $58.2+/-13.4$ years) were recruited in 12 months. Patients reported a significantly higher number of bowel movements per day than controls ( 2 versus $1, \mathrm{P}<0.001$ ). Initial analysis suggested that patients had a significantly lower total MSKCC score (group difference mean $-3.1, \mathrm{CI}-5.8$ to $-0.4, \mathrm{p}=0.02$ ), indicating worse bowel function in the patient group. Following adjustment for age, sex and co-morbidities, differences in total score were no longer significant, but the patient group had a lower score for the frequency subscale and the ability to control flatus $(p=0.008$ and $p=<0.001)$. MSKCC frequency scores were worse after right-sided resections $(p=0.03)$. High scores for complete emptying and the 